\title{
Optimization of MIMO Transceiver with Limited Feedback Channel
}

\author{
Pei Xiao ${ }^{\dagger}$, Qingchun Chen ${ }^{\S}$ \\ ${ }^{\dagger}$ Centre for Communication Systems Research (CCSR) \\ University of Surrey, Guildford, Surrey, GU2 7XH, United Kingdom \\ E-mail: p.xiao@surrey.ac.uk \\ ${ }^{\S}$ Key Lab of Information Coding and Transmission \\ Southwest Jiaotong University, Chengdu 610031, China \\ E-mail: qcchen@swjtu.edu.cn
}

\begin{abstract}
This work addresses joint transceiver optimization for multiple-input, multiple-output (MIMO) systems. In practical systems the complete knowledge of channel state information (CSI) is hardly available at transmitter. To tackle this problem, we resort to the codebook approach to precoding design, where the receiver selects a precoding matrix from a finite set of predefined precoding matrices based on the instantaneous channel condition and delivers the index of the chosen precoding matrix to the transmitter via a bandwidth-constraint feedback channel. In this paper, the codebook based precoding design at transmitter is optimized jointly with decoding design at receiver.
\end{abstract}

\section{INTRODUCTION}

MIMO systems have attracted significant interests due to the advances in wireless communication systems, aimed at satisfying the increasing demand of high bit-rate services. In MIMO systems, performance improvements can be achieved by exploiting channel state information (CSI) at the transmitter. In this case, the quality of the communication link can be improved by jointly designing the precoder and decoder. CSI can be estimated in the receiver in time division duplex (TDD) system and fedback to the transmitter. Joint design of precoding at the transmitter and equalization at the receiver for multicarrier MIMO channels under a variety of design criteria was addressed in [1], where the authors formulated the design problem within the framework of convex optimization theory, in which a number of design criteria can be easily accommodated and efficiently solved. Joint design of optimum linear precoder and decoder for a MIMO channel using a weighted minimum mean square error (MMSE) criterion subject to a transmit power constraint was treated in [2]. Closed form solutions are derived for the optimum precoder and decoder as functions of error weights, transmit power, receiver noise variance, and eigenvalues of the MIMO channel.

Most of the existing work (including those mentioned above) on joint transceiver design assumes perfect channel state information (CSI) at transmitter, which is not a realistic assumption. CSI is usually imperfect due to channel estimation errors, time-variation of channel gains, bandwidth constraint of the feedback channel, etc. To tackle this problem, we use the codebook approach to precoder design. Based on the channel information, the receiver chooses a precoding matrix from a finite codebook then convey the index of the chosen matrix to the transmitter using a limited number of bits. In the sequel, we shall discuss how this codebook based precoding and decoding can be optimized jointly.

Notations: $(\cdot)^{\mathcal{T}}$ denotes matrix transpose, $(\cdot)^{\mathcal{H}}$ matrix conjugate transpose, $(\cdot)^{*}$ matrix conjugate, $\mathrm{E}[\cdot]$ expectation, $\|\cdot\|$ Euclidian norm, trace $(\cdot)$ trace operation, and $\mathbf{I}_{N}$ an $N \times N$ identity matrix.

\section{SySTEM MODEL}

A generic MIMO communication system model is shown in Fig. 1. The input symbols streams are passed through a linear precoder optimized for a known channel. The precoder is a matrix with complex elements and can add redundancy to the input symbol streams to improve system performance. The precoder output is transmitted over the MIMO channel through $N_{t}$ transmit antennas. The signal is received by $N_{r}$ receive antennas and processed by a linear decoder, which is optimized for the fixed and known channel. The linear decoder also operates in the complex field and removes any redundancy that has been introduced by the precoder. The received signal can be expressed as

$$
\tilde{\mathbf{s}}=\mathbf{G H F s}+\mathbf{G n},
$$

where $\mathbf{H} \in \mathbb{C}^{N_{r} \times N_{t}}$ is the channel matrix, $\tilde{\mathbf{s}} \in \mathbb{C}^{M_{t} \times 1}$ is the received signal vector, $\mathbf{s} \in \mathbb{C}^{M_{t} \times 1}$ is the transmitted symbol vector, $\mathbf{n} \in \mathbb{C}^{N_{t} \times 1}$ is the noise vector, each element of which has zero mean and variance $\sigma_{n}^{2}, \mathbf{F} \in \mathbb{C}^{N_{t} \times M_{t}}$ is the precoding matrix and $\mathbf{G} \in \mathbb{C}^{M_{t} \times N_{r}}$ is the decoding matrix. The precoder adds a redundancy of $N_{t}-M_{t}$ across space since it has $M_{t}$ input symbols and $N_{t}$ precoded symbols that are transmitted simultaneously through $N_{t}$ transmit antennas. The estimate of the tranmsitted symbol vector $\hat{\mathbf{s}}$ is obtained by making hard decision on the received signal vector $\tilde{\mathbf{s}}$.

The precoding and decoding matrices $\mathbf{F}, \mathbf{G}$ are optimized according to the MMSE criterion, i.e.,

$$
\mathbf{G}, \mathbf{F}=\arg \min _{\mathbf{G}, \mathbf{F}} \mathrm{E}\left[\|\mathbf{s}-\tilde{\mathbf{s}}\|^{2}\right] .
$$

Next we present a joint precoder-decoder design using codebook and limited feedback channel. 


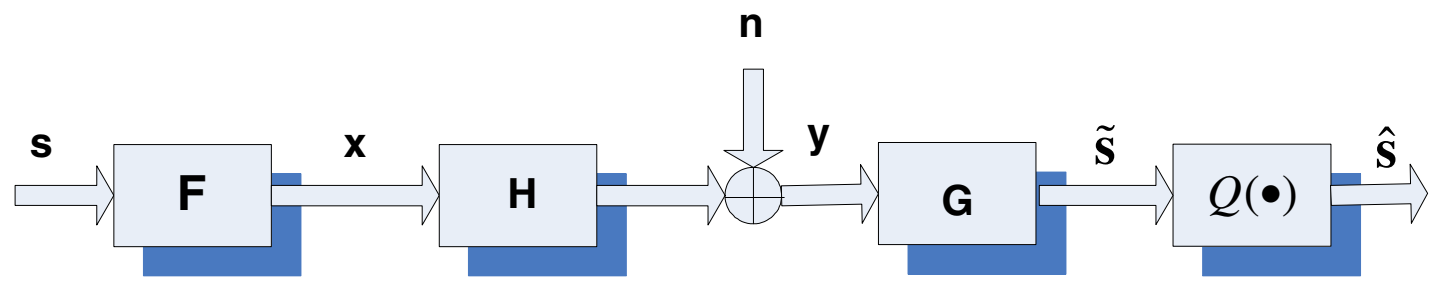

Fig. 1. Joint transmitter and receiver design.

\section{JOINT PRECODER-DECODER DESIGN}

First let us derive the decoding matrix $\mathbf{G}$ assuming the precoding matrix $\mathbf{F}$ is known. The derivation of $\mathbf{F}$ without the dependency of $\mathbf{G}$ will be given afterwards. The problem to be solved for linear MMSE receive filter is given as

$$
\begin{aligned}
\mathbf{G} & =\arg \min _{\mathbf{G}} \mathrm{E}\left[\|\mathbf{e}\|^{2}\right]=\arg \min _{\mathbf{G}} \mathrm{E}\left[\|\mathbf{s}-\tilde{\mathbf{s}}\|^{2}\right] \\
& =\arg \min _{\mathbf{G}} \mathrm{E}\left[\|\mathbf{G}(\mathbf{H F} \mathbf{s}+\mathbf{n})-\mathbf{s}\|^{2}\right],
\end{aligned}
$$

The mean square error (MSE) function can be reformed as $\mathrm{E}\left[\|\mathbf{e}\|^{2}\right]$

$=\operatorname{trace}\left\{(\mathbf{G H F} \mathbf{s}+\mathbf{G n}-\mathbf{s})\left(\mathbf{s}^{\mathcal{H}} \mathbf{F}^{\mathcal{H}} \mathbf{H}^{\mathcal{H}} \mathbf{G}^{\mathcal{H}}+[\mathbf{G n}]^{\mathcal{H}}-\mathbf{s}^{\mathcal{H}}\right)\right\}$

$=\operatorname{trace}\left\{\mathbf{G H F R}_{s} \mathbf{F}^{\mathcal{H}} \mathbf{H}^{\mathcal{H}} \mathbf{G}^{\mathcal{H}}-\mathbf{G H F R}_{s}-\mathbf{R}_{s} \mathbf{F}^{\mathcal{H}} \mathbf{H}^{\mathcal{H}} \mathbf{G}^{\mathcal{H}}\right.$

$\left.+\sigma_{n}^{2} \mathbf{G G}^{\mathcal{H}}+\mathbf{R}_{s}\right\}$,

where $\mathbf{R}_{s}=\mathrm{E}\left[\mathbf{s s}^{\mathcal{H}}\right]$. Since

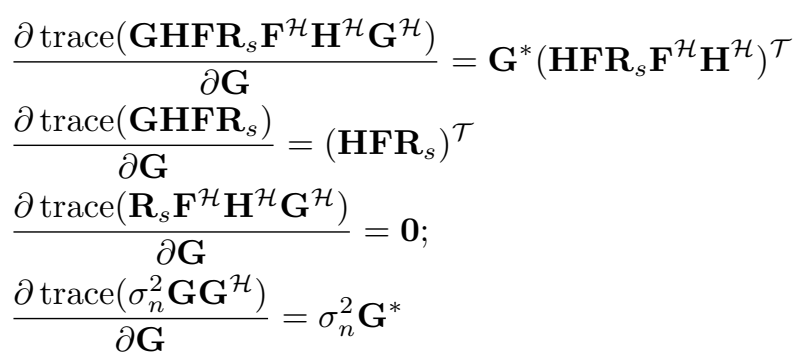

Setting the partial derivatives with respect to $\mathbf{G}$ to zero results in the matrix equation

$$
\mathbf{G}^{*}\left(\mathbf{H F R}_{s} \mathbf{F}^{\mathcal{H}} \mathbf{H}^{\mathcal{H}}\right)^{\mathcal{T}}+\sigma_{n}^{2} \mathbf{G}^{*}=(\mathbf{H F R})^{\mathcal{T}},
$$

which can be reformed as

$$
\mathbf{G}\left\{\left(\mathbf{H F R}_{s} \mathbf{F}^{\mathcal{H}} \mathbf{H}^{\mathcal{H}}\right)^{\mathcal{H}}+\sigma_{n}^{2} \mathbf{I}\right\}=\left(\mathbf{H F R}_{s}\right)^{\mathcal{H}},
$$

leading to the solution

$$
\begin{aligned}
\mathbf{G} & =\left(\mathbf{H F R}_{s}\right)^{\mathcal{H}}\left\{\left(\mathbf{H F R}_{s} \mathbf{F}^{\mathcal{H}} \mathbf{H}^{\mathcal{H}}\right)^{\mathcal{H}}+\sigma_{n}^{2} \mathbf{I}\right\}^{-1} \\
& =\mathbf{R}_{s}^{\mathcal{H}} \mathbf{F}^{\mathcal{H}} \mathbf{H}^{\mathcal{H}}\left\{\mathbf{H F R}_{s}^{\mathcal{H}} \mathbf{F}^{\mathcal{H}} \mathbf{H}^{\mathcal{H}}+\sigma_{n}^{2} \mathbf{I}\right\}^{-1}
\end{aligned}
$$

Assuming $\mathbf{R}_{s}=\mathrm{E}\left[\mathbf{s s}^{\mathcal{H}}\right]=\mathbf{I}_{M_{t}}$, i.e., the average symbol energy is normalized to unity, we have

$$
\mathbf{G}=\mathbf{F}^{\mathcal{H}} \mathbf{H}^{\mathcal{H}}\left(\mathbf{H F F}{ }^{\mathcal{H}} \mathbf{H}^{\mathcal{H}}+\sigma_{n}^{2} \mathbf{I}\right)^{-1} \text {. }
$$

Using matrix inversion lemma $\mathbf{A}^{\mathcal{H}}\left(\mathbf{A} \mathbf{A}^{\mathcal{H}}+\mathbf{I}\right)^{-1}=$ $\left(\mathbf{A}^{\mathcal{H}} \mathbf{A}+\mathbf{I}\right)^{-1} \mathbf{A}^{\mathcal{H}}$, we obtain

$$
\mathbf{G}=\left(\mathbf{F}^{\mathcal{H}} \mathbf{H}^{\mathcal{H}} \mathbf{H F}+\sigma_{n}^{2} \mathbf{I}\right)^{-1} \mathbf{F}^{\mathcal{H}} \mathbf{H}^{\mathcal{H}} \text {. }
$$

Given the knowledge of the precoding matrix $\mathbf{F}$, the decoding matrix $\mathbf{G}$ can be obtained by Eq. (4). Next, we show how the precoding matrix $\mathbf{F}$ can be derived.

According to (3),

$$
\begin{array}{r}
M S E(\mathbf{G}, \mathbf{F})=\operatorname{trace}\left\{\mathbf{G H} \mathbf{H} \mathbf{F}^{\mathcal{H}} \mathbf{H}^{\mathcal{H}} \mathbf{G}^{\mathcal{H}}-\mathbf{G H F}\right. \\
\left.-\mathbf{F}^{\mathcal{H}} \mathbf{H}^{\mathcal{H}} \mathbf{G}^{\mathcal{H}}+\sigma_{n}^{2} \mathbf{G G}^{\mathcal{H}}+\mathbf{I}\right\} \\
=\operatorname{trace}\left\{\mathbf{G}\left(\mathbf{H F F} \mathbf{F}^{\mathcal{H}} \mathbf{H}^{\mathcal{H}}+\sigma_{n}^{2} \mathbf{I}\right) \mathbf{G}^{\mathcal{H}}\right. \\
\left.-\mathbf{F}^{\mathcal{H}} \mathbf{H}^{\mathcal{H}} \mathbf{G}^{\mathcal{H}}+\mathbf{I}-\mathbf{G H F}\right\} .
\end{array}
$$

According to (4),

$$
\mathbf{G}\left(\mathbf{F}^{\mathcal{H}} \mathbf{H}^{\mathcal{H}} \mathbf{H F}+\sigma_{n}^{2} \mathbf{I}\right)=\mathbf{F}^{\mathcal{H}} \mathbf{H}^{\mathcal{H}},
$$

which is equivalent to

$$
\mathbf{G}\left(\mathbf{F}^{\mathcal{H}} \mathbf{H}^{\mathcal{H}} \mathbf{H F}+\sigma_{n}^{2} \mathbf{I}\right) \mathbf{G}^{\mathcal{H}}=\mathbf{F}^{\mathcal{H}} \mathbf{H}^{\mathcal{H}} \mathbf{G}^{\mathcal{H}} .
$$

Substituting the above equation into (5) yields

$$
\begin{aligned}
M S E(\mathbf{F}) & =\operatorname{trace}\{\mathbf{I}-\mathbf{G H F}\} \\
& =\operatorname{trace}\left\{\mathbf{I}-\left(\mathbf{F}^{\mathcal{H}} \mathbf{H}^{\mathcal{H}} \mathbf{H F}+\sigma_{n}^{2} \mathbf{I}\right)^{-1} \mathbf{F}^{\mathcal{H}} \mathbf{H}^{\mathcal{H}} \mathbf{H F}\right\}
\end{aligned}
$$

Eq. (6) can be reformed as

$$
\begin{aligned}
& M S E(\mathbf{F})=\operatorname{trace}\left\{\mathbf{I}-\left(\mathbf{F}^{\mathcal{H}} \mathbf{H}^{\mathcal{H}} \mathbf{H F}+\sigma_{n}^{2} \mathbf{I}\right)^{-1} \mathbf{F}^{\mathcal{H}} \mathbf{H}^{\mathcal{H}} \mathbf{H F}\right\} \\
& =\operatorname{trace}\left\{\mathbf{I}-\left(\mathbf{F}^{\mathcal{H}} \mathbf{H}^{\mathcal{H}} \mathbf{H F}+\sigma_{n}^{2} \mathbf{I}\right)^{-1}\left(\mathbf{F}^{\mathcal{H}} \mathbf{H}^{\mathcal{H}} \mathbf{H} \mathbf{F}+\sigma_{n}^{2} \mathbf{I}-\sigma_{n}^{2} \mathbf{I}\right)\right\} \\
& =\operatorname{trace}\left\{\mathbf{I}-\mathbf{I}+\sigma_{n}^{2}\left(\mathbf{F}^{\mathcal{H}} \mathbf{H}^{\mathcal{H}} \mathbf{H F}+\sigma_{n}^{2} \mathbf{I}\right)^{-1}\right\} \\
& =\operatorname{trace}\left\{\left(\mathbf{F}^{\mathcal{H}} \mathbf{H}^{\mathcal{H}} \mathbf{H F} / \sigma_{n}^{2}+\mathbf{I}\right)^{-1}\right\}
\end{aligned}
$$

As indicated by Eq. (7), the dependency of $\mathbf{G}$ on $\mathbf{F}$ has been removed. Now the question is how to derive the optimal precoder $\mathbf{F}^{\text {opt }}$ based on Eq. (7). Apparently, the derivation of $\mathbf{F}^{\text {opt }}$ requires the knowledge of the channel matrix $\mathbf{H}$ which can be fedback from receiver to transmitter. Much of prior work in this area was conducted based on the assumption of perfect knowledge of CSI at the transmitters. Due to the bandwidth constraint in practical wireless systems, the feedback channel is only able to communicate a finite number of bits per block. The receiver can either perform quantization on the channel matrix and feedback the quantized channel information to the transmitter; or pre-define a finite set of precoding matrices called codebook and instruct the transmitter to select the best precoder from the codebook based on the channel condition. It was discovered in [3] that the latter approach is much preferred, directly quantizing the channel 
with 16 bits of feedback performs much worse than a 6-bit feedback codebook based precoder. It was also observed in [3] that the number of feedback bits $L$ in practical systems needs not be large. Assuming perfect CSI (which is equivalent to $L=\infty$ ) does not lead to substantial gain compared to $L=6$, the limited feedback precoder obtains performance close to that of the unquantized precoder. In the sequel, we discuss precoding design with codebook approach.

\section{Codebook Based Precoder Design with Limited FEEDBACK}

For a $q$-bits feedback channel, the system needs to prepare a total of $L=2^{q}$ precoding matrices, denoted as $\mathbf{F}_{1}, \mathbf{F}_{2}, \ldots, \mathbf{F}_{L}$ and collected into a codebook $\mathcal{F}$ as $\mathcal{F}:=\left\{\mathbf{F}_{1}, \mathbf{F}_{2}, \ldots, \mathbf{F}_{L}\right\}$. Based on the current channel realization, the receiver will decide which codeword (precoder) from the codebook $\mathcal{F}$ is the most favorable and inform the transmitter to switch to that precoder by feeding back its $q$-bit codeword index. Based on the block fading channel model, channel feedback and transmitter adaptation are done on a per block basis. The codebook $\mathcal{F}$ which consists of a finite number of matrices represent a set of subspaces in the Grassmann manifold. Designing sets of $L$ matrices that maximize the minimum subspace distance (where distance can be chosen in a number of different ways, such as the chordal distance, the projection two-norm distance and the Fubini-Study distance between two subspaces [4]) is known as Grassmannian subspace packing [5]. It was observed in [3] that the performance of different codebooks are not clearly distinguishable. Hence, sticking to the codebook with any distance optimized will be comparably good. One simple method for designing good packings with arbitrary distance functions is to use the non-coherent constellation designs demonstrated in [6], which has been shown to yield codebooks with large minimum distances and can be easily modified to work with any distance function on the Grassmann manifold. This codebook design also requires the least amount of storage at both transmitter and receiver. For those reasons, we will use the structured block-circulant codebook proposed in [6] in this work. In this design, a codebook is fully specified once the first codeword $\mathbf{F}_{1}$ and a diagonal rotation matrix $\mathbf{Q}$ is provided. The other codewords in the codebook are given by

$$
\mathbf{F}_{l}=\mathbf{Q}^{l} \mathbf{F}_{l},
$$

for $l=2, \ldots, L$. The matrix $\mathbf{Q}$ is a diagonal matrix fully parameterized by an integer vector $\mathbf{u}=\left[\begin{array}{lll}u_{1} & \ldots & u_{N_{t}}\end{array}\right]$, i.e.,

$$
\mathbf{Q}=\left[\begin{array}{ccc}
\exp \left(j \frac{2 \pi}{L} u_{1}\right) & & 0 \\
0 & \cdots & \exp \left(j \frac{2 \pi}{L} u_{N_{t}}\right)
\end{array}\right]
$$

The first codeword $\mathbf{F}_{1}$ is chosen to be a $N_{t} \times M_{t}$ submatrix of the $N_{t} \times N_{t}$ DFT matrix $\mathbf{D}_{N_{t}}$ whose $(m, n)$ th element is $\left(\mathbf{D}_{N_{t}}\right)_{m, n}=\exp \left(j \frac{2 \pi}{N_{t}}(m-1)(n-1)\right)$, where $1 \leq m, n \leq$ $N_{t}$. Denoting $\mathbf{d}_{c}$ as the $c$-th column of the matrix $\mathbf{D}_{N_{t}}$, the first codeword is a collection of $M_{t}$ columns parameterized by the set of columns indices $\mathbf{c}=\left[\begin{array}{lll}c_{1} & \ldots & c_{M_{t}}\end{array}\right]$, i.e., $\mathbf{F}_{1}=$ $\left[\begin{array}{lll}\mathbf{d}_{c_{1}} & \ldots & \mathbf{d}_{c_{M_{t}}}\end{array}\right]$.

In Table I, we tabulate the choices of $\mathbf{u}$ and $\mathbf{c}$ for different transmit antenna numbers $N_{t}$ and spatially multiplexed data

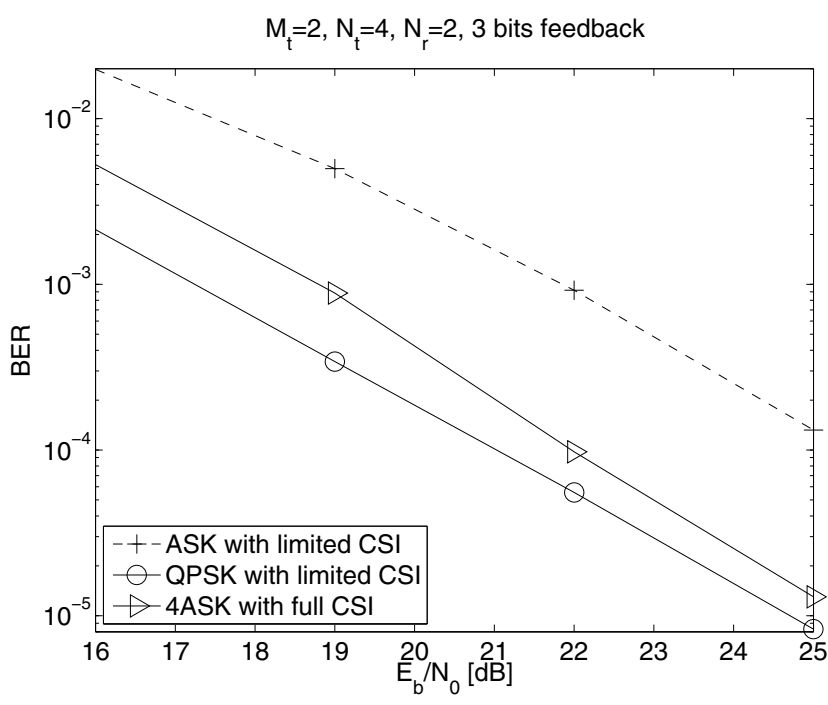

Fig. 2. Performance of the transceiver algorithm with 3-bit feedback.

stream numbers $M_{t}$. Note that the choice of $L$ is a result of trading off performance with the number of feedback bits.

Once the codebook is specified, the receiver observes a channel realization $\mathbf{H}$, selects the best precoding matrix (codeword) to be used, and feeds back the index of the codeword to the transmitter. From Section III, we know that the optimal precoder in a conventional system is chosen by minimizing MSE defined by

$$
M S E(\mathbf{F})=\operatorname{trace}\left\{\left(\mathbf{F}^{\mathcal{H}} \mathbf{H}^{\mathcal{H}} \mathbf{H F} / \sigma_{n}^{2}+\mathbf{I}\right)^{-1}\right\} .
$$

Substituting $\mathbf{F}$ with $\mathbf{F}_{l}$ in the above equation (where $\mathbf{F}_{l}$ is the $l$ th codeword in the codebook), the index of the precoding matrix to be conveyed from the receiver to the transmitter is selected as

$$
\begin{aligned}
l^{\mathrm{opt}} & =\arg \min _{l \in\{1,2, \ldots, L\}} \operatorname{MSE}\left(\mathbf{F}_{l}\right) \\
& =\arg \min _{l \in\{1,2, \ldots, L\}} \operatorname{trace}\left\{\left(\mathbf{F}_{l}^{\mathcal{H}} \mathbf{H}^{\mathcal{H}} \mathbf{H} \mathbf{F}_{l} / \sigma_{n}^{2}+\mathbf{I}\right)^{-1}\right\} .
\end{aligned}
$$

Once the precoding matrix $\mathbf{F}$ is determined, the receiver uses Eq. (4) to calculate the equalization matrix $\mathbf{G}$.

\section{A. Simulation Results}

The proposed tranceiver algorithm is applied to a $4 \times 2$ MIMO system $\left(N_{t}=4, N_{r}=2\right)$. The number of transmitted symbol streams is set to $M_{t}=2$, and the transmit power is $M_{t} \sigma_{s}^{2}=2$, i.e., unit average transmit power for each transmitted symbol. We assume uncorrelated Rayleigh fading channel and the channel matrix is normalized such that $\mathrm{E}\left[\|\mathbf{H}\|_{F}^{2}\right]=1$. The simulation results are averaged over many channel realizations. Figs. 2 and 3 show the performance comparison of different systems with 3-bit and 6-bit feedback, respectively. The employed modulation schemes are 4ASK, QPSK, which are chosen such that all the systems have the same data transmission rate or spectrum efficiency. As expected, the QPSK system performs much better than the 4ASK system. Comparing Fig. 2 to Fig. 3, it is evident that the system performance improves as the number of feedback bits 
TABLE I

PARAMETERS FOR $\mathbf{F}_{1}$ AND $\mathbf{Q}$

\begin{tabular}{|c|c|c|c|c|c|c|c|c|}
\hline$N_{t}$ & $M_{t}$ & $L / q=\log _{2} L$ & $\mathbf{c}=$ & $c_{1} \quad \ldots$ & $c_{M_{t}}$ & $\overline{\mathbf{u}=}$ & $u_{1} \quad \ldots$ & $u_{N}$ \\
\hline 2 & 1 & $8 / 3$ & & [1] & & & $0]$ & \\
\hline 3 & 1 & $32 / 5$ & & [1] & & & $\begin{array}{ll}1 & 26\end{array}$ & $28]$ \\
\hline 3 & 2 & $32 / 5$ & & {$\left[\begin{array}{ll}1 & 2\end{array}\right]$} & & & $\begin{array}{ll}1 & 26\end{array}$ & 28 \\
\hline 4 & 1 & $64 / 6$ & & \begin{tabular}{|l|l|}
1 &
\end{tabular} & & 1 & $8 \quad 61$ & 45 \\
\hline 4 & 2 & $64 / 6$ & & {$\left[\begin{array}{ll}0 & 1\end{array}\right]$} & & 1 & $\begin{array}{ll}7 & 52\end{array}$ & 56 \\
\hline 4 & 3 & $64 / 6$ & & 0 & & 1 & 861 & 45 \\
\hline
\end{tabular}

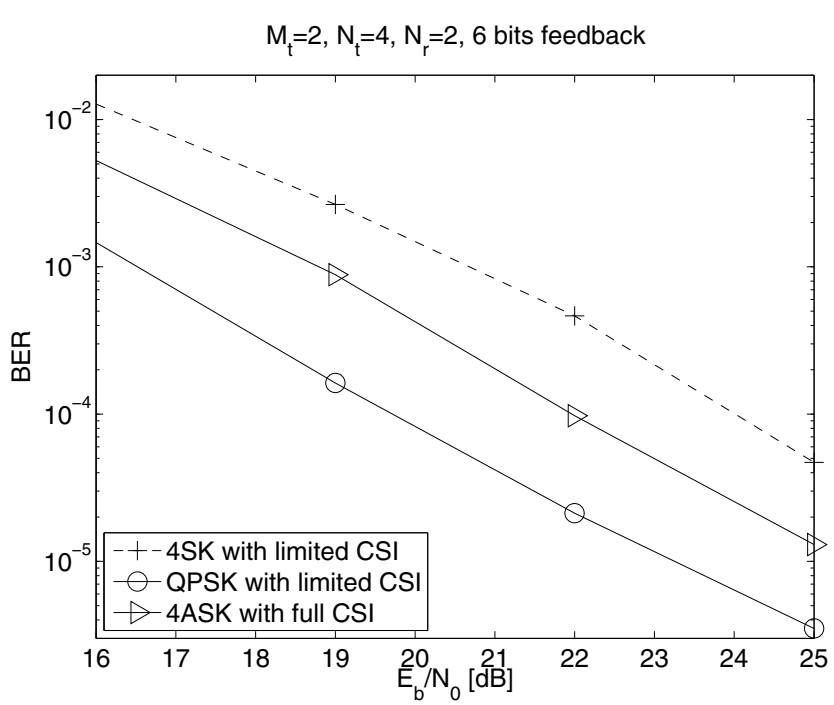

Fig. 3. Performance of the transceiver algorithm with 6-bit feedback.

increases. For example, the 4ASK system with 6-bit feedback has closer performance to the system with full CSI compared to that with 3-bit feedback.

\section{CONCLUSions}

We have presented solution for joint precoding and decoding optimization for MIMO systems with limited feedback channel. In our design, only a few bits are needed to carry the index of the selected precoding matrix (codeword) in a codebook from receiver to transmitter. The simulation results show that the performance of the proposed system with limited CSI can approach that with full CSI given sufficient number of the feedback bits.

\section{ACKNOWLEDGEMENT}

Qingchun Chen's work is partly supported by the NSFC under grant no. 60872013 and the the Fundamental Research Funds for the Central Universities under grant no. SWJTU11CX043.

\section{REFERENCES}

[1] D. Palomar, J. Cioffi, M. Lagunas. "Joint Tx-Rx beamforming design for multicarrier MIMO channels: a unified framework for convex optimization", IEEE Trans. Signal Proc., vol. 51, no. 9, pp. 2381-2401, Sept. 2003.

[2] H. Sampath, P. Stoica, A. Paulraj. "Generalized linear precoder and decoder design for MIMO channels using the weighted MMSE criterion", IEEE Trans. Commun., vol. 49, no. 12, pp. 2198-2206, Dec. 2001.
[3] S. Zhou, B. Li "BER criterion and codebook construction for finite-rate precoded spatial multiplexing with linear receivers," IEEE Trans. Sig. Proc., vol. 54, no. 5, pp. 1653-1664, May 2006.

[4] D. Love, R. Heath. "Limited feedback unitary precoding for spatial multiplexing systems," IEEE Trans. on Inform. Theory, vol. 51, no. 8, pp. 2967-2976, August 2005.

[5] A. Barg, D. Nogin. "Bounds on packings of spheres in the Grassmann manifold," IEEE Trans. on Inform. Theory, vol. 48, no. 9, pp. 24502454, Sept. 2002.

[6] B. Hochwald, T. Marzetta, T. Richardson, W. Sweldens, R. Urbanke. "Systematic design of unitary space-time constellation", IEEE Trans. on Inform. Theory, vol. 46, no. 6 pp. 1962-1973, Sept. 2000. 